\title{
Die dritte Botschaft aus dem Jenseits lässt Erwartungen offen
}

Isabel Zwyssig

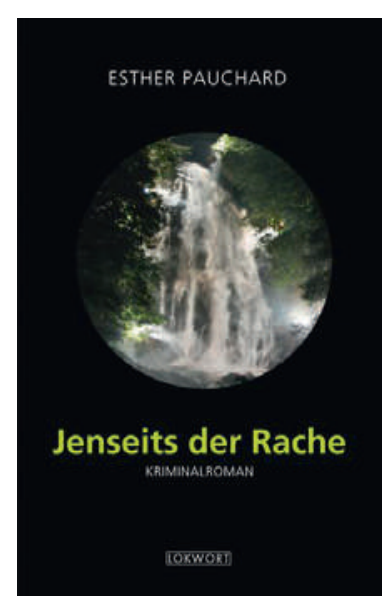

Esther Pauchard Jenseits der Rache

Bern: Lokwort Verlag; 2014. 336 Seiten. 34 CHF. ISBN 978-3-906786-55-1
Mit spitzer Feder überschreitet sie Grenzen. Esther Pauchard, Autorin und Fachärztin für Psychiatrie und Psychotherapie aus Thun, verschont weder die Charaktere in ihren Kriminalromanen noch die geneigte Leserschaft ausserhalb der fiktiven Welt vor plötzlichen Perspektivenwechseln, Vertrauensbrüchen und - speziell im dritten Fall - Wasserleichen. Nach Jenseits der Couch (2010) und Jenseits der Mauern (2012) - beide herausgegeben vom Nydegg Verlag Bern - ist im Herbst dieses Jahres im Berner Lokwort Verlag Jenseits der Rache erschienen. Wie bereits in den vorangegangenen Werken hat Esther Pauchard in ihrem dritten kriminalistischen Streich ihre Erfahrungen aus dem psychiatrischen Berufsalltag geschickt einfliessen lassen. Wieder treiben seelische Abgründe Menschen zu Taten, die man ihnen auf den ersten Blick nicht zutrauen würde.

\section{Ein Starpsychoanalytiker wird zur Wasserleiche}

Tiefer Fall und Abgründe spielen in Jenseits der Rache im ganz wörtlichen Sinn eine zentrale Rolle. Als Kassandra «Ka» Bergen, Oberärztin an der Psychiatrischen Klinik Eschenberg und Mutter, mit ihrem Ehemann Marc, Hausarzt, und einem befreundeten Paar, dem Psychiater Martin und seiner Partnerin Selma, Psychologin, ein Wochenende im edlen Grand Hotel Giessbach verbringt, ereignet sich Rätselhaftes. Der Berner Starpsychoanalytiker Adrian Wyss stürzt in den Giessbachfällen zu Tode. Handelt es sich dabei wirklich um einen Selbstmord, wie zunächst von behördlicher Seite und dem Umfeld des Verstorbenen angenommen? Ka Bergen und ihre Begleiter sind skeptisch. Es deutet alles darauf hin, dass der plötzliche Tod des gefeierten, weltmännischen Psychiaters mit anderen Todesfällen in Zusammenhang steht, deren Hergang nie mit letzter Sicherheit geklärt werden konnte. Bei den Nachforschungen gerät vor allem Ka aufgrund ihres stark ausgeprägten Miss-Marple-Spürsinns schneller unter Verdacht, als ihr lieb ist. Warum ruft Adrian Wyss sie kurz vor seinem Sturz in die Wassermassen auf ihrem Mobiltelefon an? Weshalb wird ihre Patientin Marie Lanz nachhaltig erschüttert, als sie von Wyss' Hinschied erfährt, bei dem sie vor ihrer Einweisung in die Psychiatrische Klinik Eschenberg ambulant behandelt worden war? War Emil Lüscher, Praxispartner und einst Mentor seines verstorbenen Kollegen, neidisch auf dessen berufliche Erfolge? Startherapeut Wyss galt als wichtiges Bindeglied in der Leitung der umstrittenen INTPERS-Studie über internetbasierte Psychotherapie für Borderlinepatienten. Auch Ange- hörige aus der Chefetage der Klinik Eschenberg sind hier involviert. Führt eine heisse Spur gar an den Arbeitsplatz von Ka und ihren Freunden Martin und Selma? Bald kommen sie über die unnahbare, hochambitionierte Claudine Mathier, Chief Operating Officer bei einem Basler Pharmaunternehmen, ins Grübeln.

\section{Jenseits des Gewöhnlichen}

Pauchards Charaktere und Handlungen bewegen sich stets jenseits allgemein gültiger gesellschaftlicher und ethischer Normen, oft aber auch jenseits der Lesererwartung. Hier macht Jenseits der Rache keine Ausnahme. Ka Bergen setzt ihre Stellung als Oberärztin mutwillig aufs Spiel, als sie sich ins Büro von Klinikdirektor Rudolf Blanc schleicht, um in seinen Daten Hinweise darauf zu finden, ob möglicherweise ein Zusammenhang zwischen dem Tod von Adrian Wyss und der kontroversen INTPERS-Studie besteht. Prompt wird sie beim Schnüffeln erwischt und von ihrem Dienst freigestellt. Auf einer detektivischen Undercover-Mission gibt sich Kas Cousine Nora in der Praxis von Emil Lüscher als spirituell erleuchtete Patientin aus, die seltsame Botschaften aus der geistigen Welt empfängt. Solche Kapriolen aus der Pauchard'schen Feder muten zweifellos amüsant an und treiben den scheinbar bodenständigen Facharzt, der teilweise wie sein verstorbener Kollege in einem unrühmlichen Abhängigkeitsverhältnis zu seinen Patientinnen steht, zu Verzweiflungstaten.

\section{Wo sind die kriminalistischen Irrpfade?}

Eine Wendung war für Kenner der Pauchard'schen Kriminalromane durchaus vorhersehbar: Ka verbringt mit ihrem langjährigen besten Freund und Arbeitskollegen Martin eine Liebesnacht. Spannend, gut konstruiert, manchmal realitätsfern und dann wieder erstaunlich lebensnah sind die bisweilen etwas zu weit verzweigten Handlungsstränge in Jenseits der Rache. Immer stellen sie das Menschliche mit viel Wortwitz in den Mittelpunkt. Grosse Überraschungen, wie man sie etwa aus Jenseits der Mauern kennt, bleiben - Ausnahme bildet die Brandstiftung im Heim von Familie Bergen - aber aus. Geschickt angelegte, konzeptuelle Fallstricke, in denen man sich als Lesende beim Rätseln so gern verheddert, fehlen im dritten Roman. Vielleicht hätte etwas mehr rechtsmedizinischer Einfluss dem abhelfen können? Gestorben wird hier nicht durch die Hand eines kaltblütigen, berechnenden Mörders, sondern durch unglücklich aneinandergereihte Zufälle. Botschaften aus dem Jenseits? 